\title{
Muscle Activation Strategies and Symmetry of Spinal Loading in the Lumbar Spine With Scoliosis
}

\author{
Ian A.F. Stokes, PhD and Mack Gardner-Morse, MS
}

Study Design. Biomechanical analysis of muscle and spinal forces in a lumbar spine with scoliosis.

Objectives. To calculate spinal loading asymmetry and its dependence on muscle activation strategy.

Summary of Background Data. It is commonly assumed that a spine with scoliosis experiences greater loading on the concave side and that this asymmetric loading causes asymmetric growth and progression of deformity. However, neither the magnitude of the asymmetric loading imposed on the spine as a function of the scoliosis curve nor the resulting mechanically altered vertebral growth and disc remodeling have been quantified.

Methods. Spinal loading was estimated in a lumbar spine model with increasing degrees of scoliosis. External loading was each of three pure moments or forces acting at T12, with magnitudes of either $50 \%$ or $75 \%$ of maximum effort. For each external loading, the muscle activation patterns were determined with each of three different muscle activation strategies in an optimization model: 1) minimize the sum of cubed muscle stresses; 2 ) minimize spinal asymmetric load (i.e., "follower load"); and 3) reverse the spinal load asymmetry (increased compression on convex side) at the level of the apex.

Results. The first strategy produced loading that tended to increase the curve magnitude, with the resultant force acting at up to $15 \mathrm{~mm}$ lateral to the intervertebral disc center. Both Strategies 2 and 3 had increased muscle stress averaging between $42 \%$ and $75 \%$.

Conclusions. We speculate that individuals with scoliosis can adopt different muscle activation strategies and that these strategies may determine whether or not the spinal loading causes scoliosis progression during growth. Muscle activation patterns generating spinal loading that does not promote curve progression during growth have greater physiologic cost.

Key words: scoliosis, biomechanics, muscles, deformity. Spine 2004;29:2103-2107

Progression of scoliosis deformity during growth is thought to be associated with asymmetric loading of the spine that produces asymmetric growth. It is assumed that a spine that is straight in the coronal plane is habitually loaded symmetrically, whereas a spine with scoliosis is asymmetrically loaded. Further, there is speculation

From the Department of Orthopaedics and Rehabilitation, University of Vermont, Burlington, VT.

Acknowledgment date: July 31, 2003. Acceptance date: October 30, 2003.

Supported by NIH R01 AR 44119 and NIH R01 AR 46543.

The manuscript submitted does not contain information about medical device(s)/drug(s).

Federal funds were received in support of this work. No benefits in any form have been or will be received from a commercial party related directly or indirectly to the subject of this manuscript.

Address correspondence and reprint requests to Ian A.F. Stokes, $\mathrm{PhD}$, Department of Orthopaedics and Rehabilitation, University of Vermont, Burlington, VT 05405-0084; E-mail: Ian.Stokes@uvm.edu that the asymmetric loading causes progressive deformity, especially while the spine continues to grow. The "vicious cycle" theory of scoliosis progression ${ }^{1-3}$ proposes that scoliosis causes loading of the spine that is asymmetric in the coronal plane, and that vertebral growth and disc remodeling respond to the chronic presence of these asymmetric forces. Progression of scoliosis is observed to occur when there is rapid growth of the spine. ${ }^{4}$ This notion that progression of spinal deformity results from mechanical modulation of growth that in turn produces asymmetrically wedged vertebrae and discs is intuitively attractive, and it provides a supposed rationale for scoliosis management with braces.

While qualitatively plausible, the vicious cycle theory lacks quantification of two crucial components: the magnitude of the loading distribution across discs and vertebral growth plates, and the growth and remodeling response to mechanical loading of the vertebrae and intervertebral discs. This paper focuses on quantifying the first of these presently qualitative elements.

Direct measurement of the loading asymmetry in the spine during functional activities has not been attempted because of the lack of instrumentation capable of providing this information in live humans with scoliosis. Therefore, mathematical modeling was used in this study to estimate lumbar spinal loading. Estimates of the spinal loading and muscle activation were performed for differing magnitudes and directions of external effort, and for differing degrees of scoliosis. The biomechanical problem of estimating spinal forces is indeterminate because of the redundant number of muscles. The trunk muscle activation strategy in persons with and without scoliosis is not known. In these simulations, we used an optimization approach in which the muscle activation strategy could control the degree of asymmetry of vertebral loading, subject to bounds on muscle force magnitude and constraints on the intervertebral displacements.

The goals of this study were as follows: 1) to find the plausible range of the spinal loading asymmetry in a spine with scoliosis; 2) to find whether there are muscle activation strategies that can produce symmetric frontalplane loading of the spine with scoliosis, or loading that might tend to reverse the deformity; and 3) to investigate the physiologic costs of differing muscle activation strategies in terms of muscle forces and the spinal loading.

\section{Methods}

A previously reported mathematical model of the lumbar spine and its musculature ${ }^{5,6}$ was used as the basis for this study. The spinal geometry was modified to represent five different magnitudes of scoliosis deformity (Figure 1). The model included 


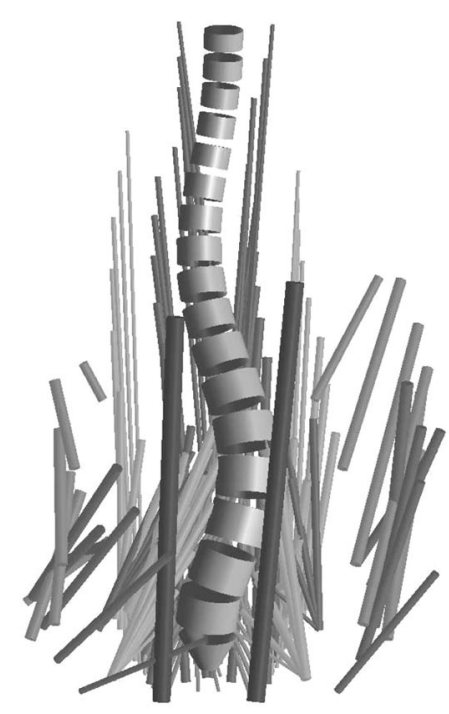

AP View

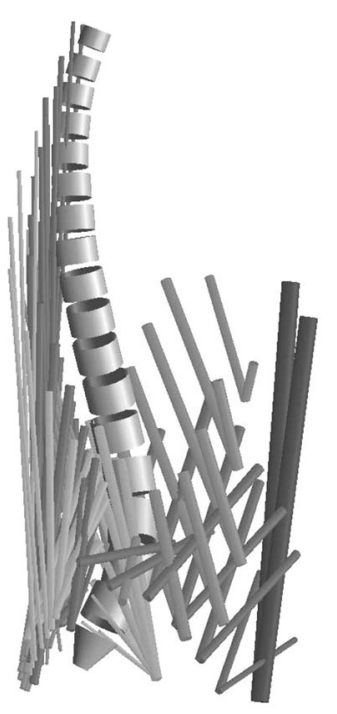

Lateral View

Figure 1. Model geometry (vertebrae and lines of action of muscles) for the model that represented a lumbar scoliosis magnitude of $38^{\circ}$ Cobb, apex at L2-L3.

six motion segments of the lumbar spine (T12-L1 through L5-S1), and the muscles that cross those levels. The thoracic spine was not included, in order to avoid additional variables associated with the ribs.

The spinal and muscular geometry was based on that reported by Stokes and Gardner-Morse. ${ }^{6}$ The initial spinal geometry (with sagittal plane curvature, but no scoliosis) was then transformed in order to produce five idealized scoliosis curves with increasing scoliosis magnitudes of $13^{\circ}, 26^{\circ}, 38^{\circ}$, and $51^{\circ}$ Cobb angle, apex at L2 as was done by Stokes. ${ }^{2}$ For each deformed spine shape, the muscle end points were repositioned according to displacements and rotations of their associated vertebral attachment sites. It was assumed that each muscle's force-generating capacity was not altered by its altered length. Similarly, it was assumed that the flexibility properties of each motion segment were not altered in a deformed position.

The intervertebral motion segments were represented as "equivalent structures" consisting of a parallel beam and a truss with a rigid offset between them. ${ }^{7}$ The stiffness values of this structure were obtained from stiffness measurements of adult human lumbar motion segments, tested with an axial preload of 500 N. ${ }^{7,8}$

The loading of the spine was analyzed for each of 12 external loading states in turn: positive and negative forces acting at T12 in each of the three principal directions relative to the global axis system, and positive and negative values of each of the three pure moments acting at T12. To obtain an initial reference for each external loading direction, the maximum possible effort for each loading direction was first calculated. These maximum effort conditions were obtained by an optimization calculation in which the objective function was to maximize the external force or moment. ${ }^{5}$ These maximum efforts were calculated for each geometry representing increasing scoliosis deformity. Subsequently, the maximum effort values obtained at the maximum deformed geometry $\left(51^{\circ}\right.$ Cobb angle) were used as the basis for subsequent simulations, since the maximum efforts at this maximally deformed geometry were found to be the lowest.

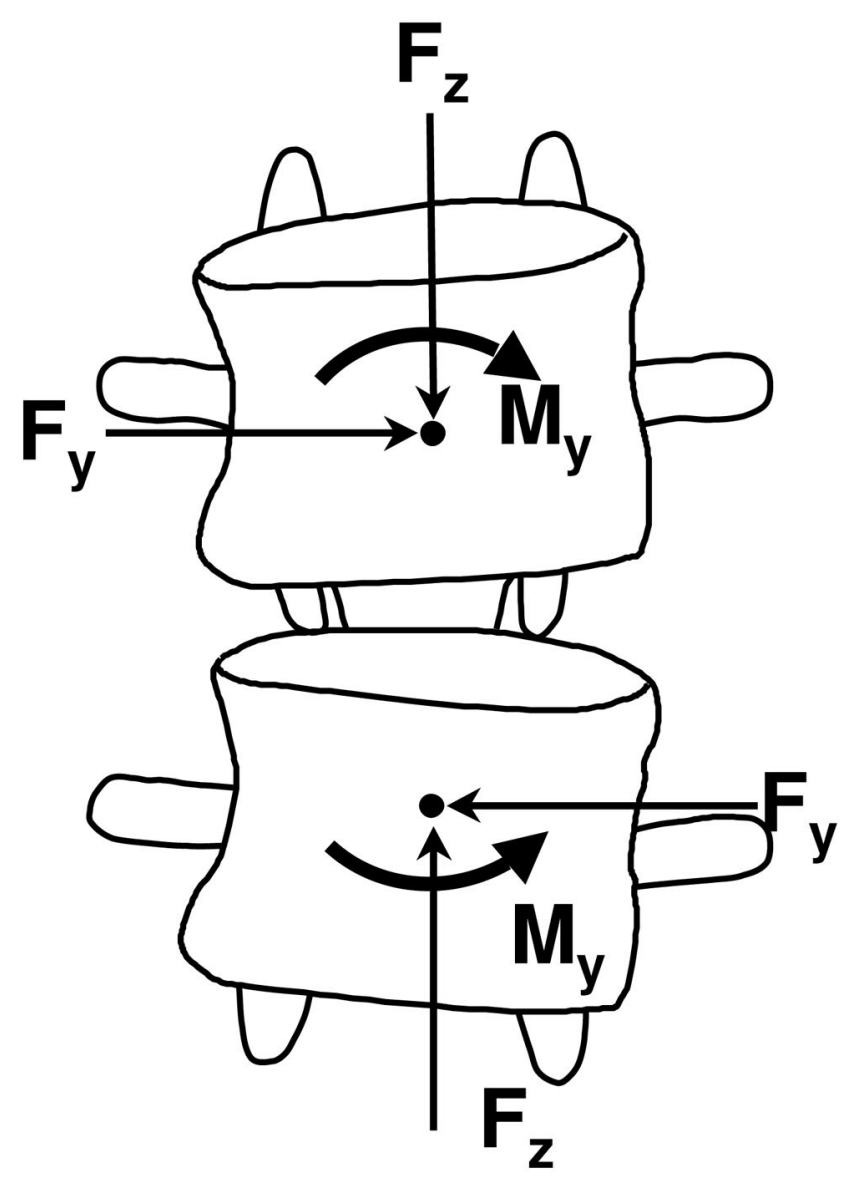

Figure 2. Components of forces acting on a spinal motion segment in the frontal plane.

The subsequent simulations in each load direction were made for two load magnitudes: $50 \%$ and $75 \%$ of these maximum efforts. At each level of effort, simulations were performed for each of three different muscle activation strategies, represented by objective functions in the optimization calculations:

Strategy 1. Minimize muscle stress: This strategy had the objective to minimize $\Sigma \sigma_{\mathrm{m}}{ }^{3}$, where $\sigma_{\mathrm{m}}$ are the 180 muscle stresses. This strategy is thought to be compatible with maximizing the muscular endurance and provides a close match to empirical EMG data. ${ }^{9}$ However, it does not take the loading of the spine into account.

Strategy 2. Follower load: Here, the objective was to minimize the sum of the squares of the lateral bending moments $\left(\mathrm{M}_{\mathrm{y}}\right.$ in Figure 2) at the midpoints between vertebral centers in all six motion segments of the lumbar spine, in addition to minimizing the sum of cubed muscle stresses $\left(\Sigma \sigma_{\mathrm{m}}{ }^{3}\right)$. The moments were expressed in $\mathrm{Nm}$, and the muscle stresses in $\mathrm{MPa}$. If this moment objective is met, the loading distribution at the intervertebral discs in the frontal plane can be considered to be uniform (similar to the follower load in the sagittal plane, as described by Patwardhan et $a l^{10}$ ).

Strategy 3. Curve correction: Maximize "self-correcting" lateral bend moments $\mathrm{M}_{\mathrm{y}}$ on motion segments above and below the curve apex (L1-L2 and L2-L3) and Min $\Sigma \sigma_{\mathrm{m}}{ }^{3}$. The moments were expressed in $\mathrm{Nmm}$, and the muscle stresses in $\mathrm{MPa}$. 
Table 1. Calculated Maximum Efforts

\begin{tabular}{lrr}
\hline & $0^{\circ}$ Cobb & $51^{\circ}$ Cobb \\
\hline Moments (Nm) & & \\
Right lateral bend & 150 & 102 \\
Flexion & 195 & 119 \\
Right axial rotation & 85 & 22 \\
Left lateral bend & 150 & 102 \\
Extension & 102 & 75 \\
Left axial rotation & 85 & 77 \\
Forces (N) & & \\
Push forward & 907 & 537 \\
Push left & 883 & 552 \\
Push up & 3643 & 1347 \\
Push backwards & 888 & 707 \\
Push right & 883 & 805 \\
Push down & 8986 & 4721 \\
\hline
\end{tabular}

In all simulations, bounds were placed on muscle forces and intervertebral displacements according to physiologically plausible limiting values, as in Stokes et al. ${ }^{5}$ The muscle stress bounds were $0<\sigma_{\mathrm{m}}<460 \mathrm{kPa}$; for intervertebral displacements, the bounds were $\pm 2 \mathrm{~mm}$ lateral shear, $\pm 5 \mathrm{~mm}$ AP shear, $5^{\circ}$ flexion and extension, and $\pm 2^{\circ}$ for lateral bend and axial rotation.

Calculations of unknown muscle forces for each of the loading directions, and each of the three muscle activation strategies (and subject to the above bounds), were performed using the MINOS optimization program (Operation Research Laboratory, Stanford University, Stanford, CA).

In evaluating the spinal loading, the force offset at each motion segment was defined as the lateral bending moment divided by the axial force magnitude $\mathrm{M}_{\mathrm{y}} / \mathrm{F}_{\mathrm{z}}$ (Figure 2). The physiologic cost under each external loading and muscle activation strategy was defined as the muscle stress averaged over the 180 muscles in the model. The intervertebral compressive force averaged over the six motion segments was also calculated.

\section{- Results}

The maximum external moments calculated for the spine without scoliosis $\left(0^{\circ}\right.$ Cobb angle) were 102 and $195 \mathrm{Nm}$ (flexion and extension), $150 \mathrm{Nm}$ lateral bending, and 85 $\mathrm{Nm}$ axial rotation. The maximum forces were $907 \mathrm{~N}$ (push forwards), $888 \mathrm{~N}$ (push back), $883 \mathrm{~N}$ (lateral force), 3643 (push up), and $8986 \mathrm{~N}$ (push down). All these maximum efforts were reduced for the spine with $51^{\circ} \mathrm{Cobb}$ scoliosis (Table 1). Subsequent analyses were made for efforts that were $50 \%$ and $75 \%$ of the maximum values calculated for the $51^{\circ} \mathrm{Cobb}$ configuration. The greatest reduction in effort for the spine with the $51^{\circ}$ scoliosis was for the right axial rotation effort (effort reduced from 85 to 22 $\mathrm{Nm})$. Since the effort was so much reduced for right axial rotation, there was no suitable basis for comparison of the submaximal efforts, so this effort direction was excluded from the subsequent analyses.

At $50 \%$ and $75 \%$ of the maximum efforts, Strategy 1 (minimize muscle stresses) predicted spinal loading whose offset from the intervertebral disc center moved toward the concave side of the scoliosis curve with increasing Cobb angle (Figure 3). This load offset was to
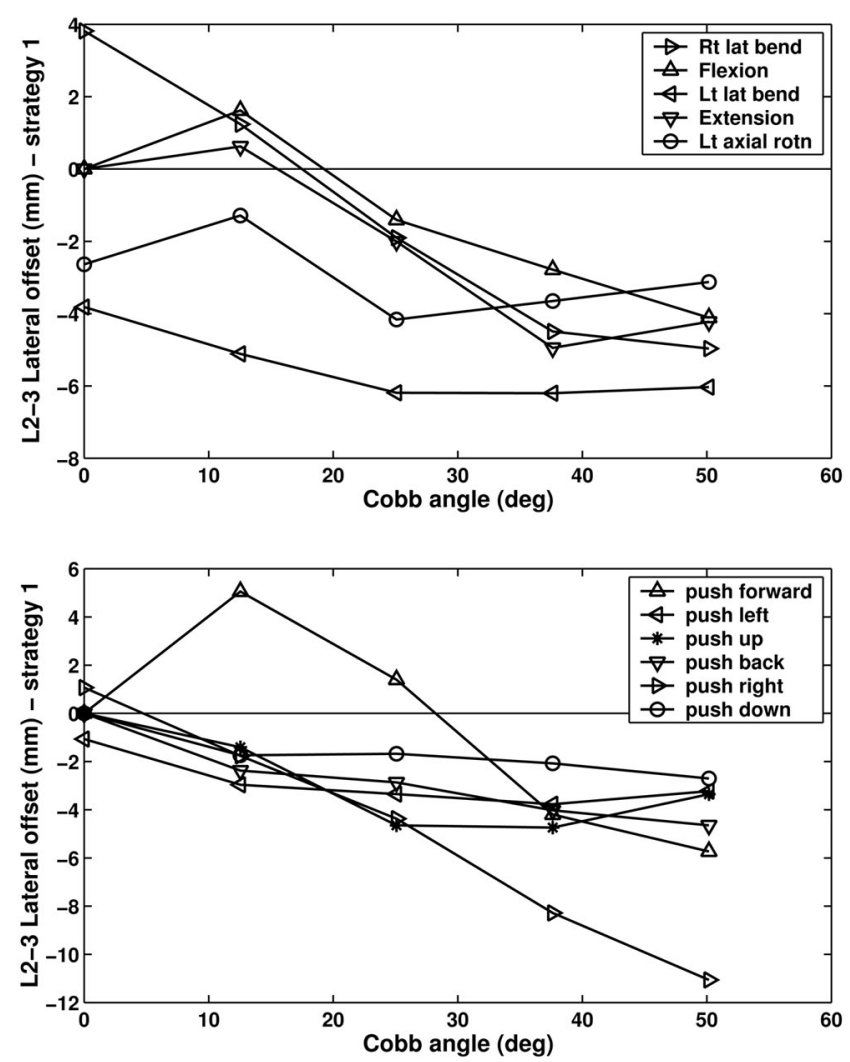

Figure 3. Force offset at L2-L3 (level below scoliosis curve apex) for five external moments (upper panel) and six force directions (lower panel) as a function of the Cobb angle.

the concave side of the vertebrae at the curve apex, compatible with growth modulation that would increase the scoliosis according to the vicious cycle theory. At $50 \%$ effort, for the $51^{\circ} \mathrm{Cobb}$ angle, the offset was $-3.9 \mathrm{~mm}$ at L1-L2 and $-6.1 \mathrm{~mm}$ at L2-L3, averaged over the 11 efforts that were considered. The corresponding offsets at $75 \%$ effort were $-5.2 \mathrm{~mm}$ and $-4.8 \mathrm{~mm}$.

The follower load strategy (Strategy 2) predicted zero or minimal intervertebral lateral bending moments (and hence offsets) in all loading cases. However, the muscle recruitment pattern that achieved this follower load state required greater muscle stresses relative to Strategy 1 (Figure 4), and correspondingly greater intervertebral compression force magnitudes (Table 2). The mean increase in muscle stress, relative to Strategy 1, was $45 \%$ at $50 \%$ effort, and $42 \%$ at $75 \%$ effort. (These averages exclude values for the "push up" effort, since the muscle forces for this effort were in opposition to the resisted external force.)

With the curve correction strategy (Strategy 3), a correcting moment was achieved under all simulated conditions at the apex, and this was associated with asymmetric loading of the vertebrae that would reverse the vicious cycle. At $50 \%$ effort, for the $51^{\circ}$ Cobb angle, the offset was $10.7 \mathrm{~mm}$ at L1-L2 and $7.2 \mathrm{~mm}$ at L2-L3, averaged over the 11 efforts that were considered. The corresponding offsets at $75 \%$ effort were $5.1 \mathrm{~mm}$ and $4.1 \mathrm{~mm}$. Again, to achieve this objective, the mean muscle stresses 

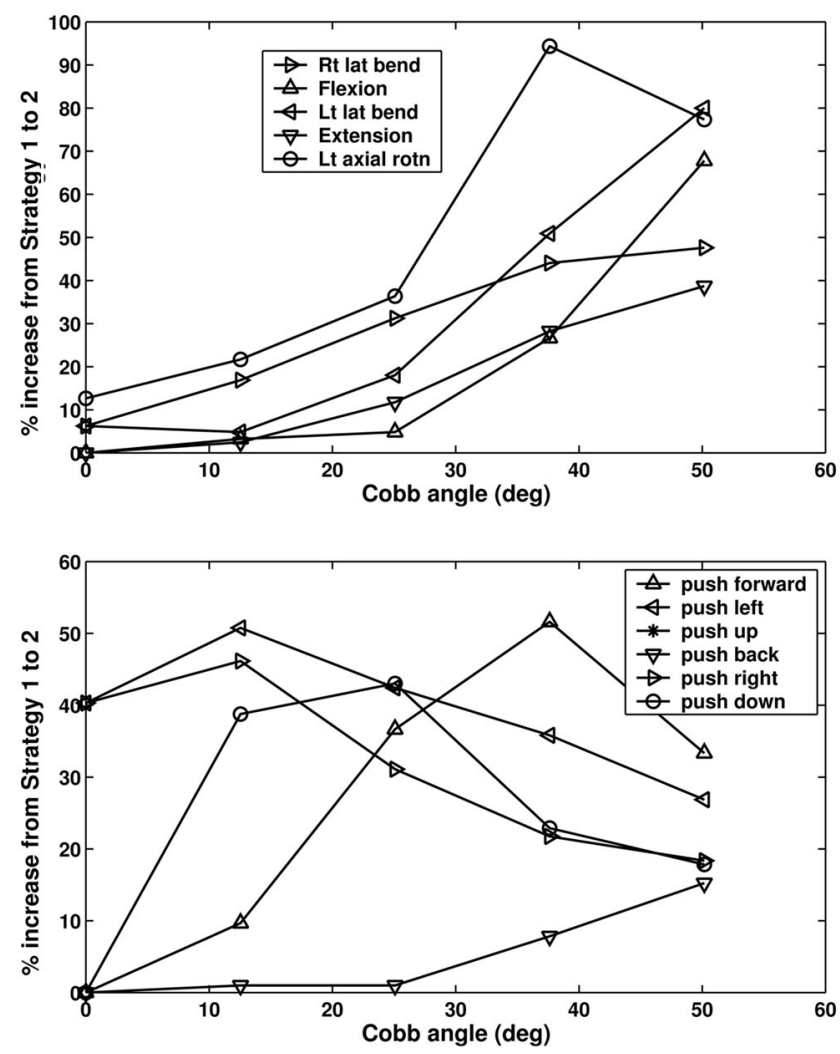

Figure 4. The increase (percent) in mean muscle stress that was required to achieve a "follower" loading of the spine (Strategy 2) relative to that for Strategy 1 (which minimized muscle stress only) for 10 different external efforts. Upper panel: moment-generating efforts. Lower panel: force-generating efforts.

were increased relative to Strategy 1 . The increase averaged $46 \%$ at $50 \%$ effort and $48 \%$ at $75 \%$ effort, similar to the increases for Strategy 2. As in Strategy 2, the associated spinal forces (Table 2) were also increased.

\section{Discussion}

The strategy that only minimized cubed muscle stresses produced vertebral and disc loading asymmetry that would increase the scoliosis, according to the calculated offsets of the resultant forces acting though motion segments. The follower load strategy that minimized the

\section{Table 2. Intervertebral Compression Force (Averaged Over Six Motion Segments) at $75 \%$ Effort for the $51^{\circ}$ Cobb Spinal Geometry}

\begin{tabular}{lrrr}
\hline Effort & Strategy 1 & Strategy 2 & Strategy 3 \\
\hline Right lateral bend & 1133.6 & 1455.9 & 1296.5 \\
Flexion & 908.0 & 1162.5 & 1418.9 \\
Left lateral bend & 841.5 & 1155.8 & 1389.9 \\
Extension & 1171.2 & 1411.3 & 1601.1 \\
Left axial rotation & 841.3 & 1319.5 & 1372.5 \\
Push forward & 657.7 & 779.3 & 800.4 \\
Push left & 885.6 & 843.6 & 946.1 \\
Push up & 1167.9 & 1401.1 & 1577.5 \\
Push back & 1209.1 & 1252.8 & 1313.3 \\
Push right & 554.1 & 594.9 & 738.3 \\
Push down & -1115.4 & -737.7 & -962.5 \\
\hline
\end{tabular}

magnitude of the intervertebral lateral bending moments was able to reduce these moments to zero, thereby creating a loading state that would produce no scoliosis progression according to the vicious cycle theory. With the follower load, ${ }^{10}$ the load takes the same path relative to the vertebrae as the spine deforms, and the follower loading imposes minimal or zero moments about the spinal motion segments. It was also possible to simulate a muscle activation strategy (curve correction strategy) that would produce a correction moment and a loading asymmetry at the apex, compatible with reversing the presumed vicious cycle mechanism of curve progression. Strategies 2 and 3 that prevented or reversed the loads that would cause deformity progression had physiologic costs, associated with the additional spinal loads and muscle forces. The spinal force was generally more for Strategy 3 than Strategy 2 (Table 2). Strategy 2 specified that the force offset at all anatomic levels be zero, whereas Strategy 3 reversed the force offset at two levels (the levels above and below the curve apex).

Several limitations of this study were associated with simplifications and assumptions made in the modeling analysis. Only the lumbar region was considered, to avoid complexities and lack of anatomic data for the thoracic region. The model representation of the motion segment behavior was based on in vitro studies of adult spines without scoliosis, and the elastic and structural behavior is probably altered by scoliosis deformity. Similarly, the analysis did not take into account the possible adaptive changes in muscles associated with their altered length and possible cross-sectional alterations in persons with scoliosis.

Several studies of the intervertebral discs in spines with scoliosis indicate that there are alterations that may be a result of asymmetric mechanical loading. Perié et $a l^{11}$ reported a displacement of the nucleus toward the convex side of the curve, based on MRI imaging. Tissue composition and cell density differences between the concave and convex sides of apical discs ${ }^{12}$ and altered protein synthesis rates ${ }^{13}$ are thought to be a result of abnormal loading, but factors such as compromised metabolite and nutrient transport also contribute to reduced cell numbers and viability. ${ }^{14,15}$

Confirmation of the muscle activation patterns predicted by these modeling analyses is complicated by the difficulties of inferring muscle forces from electromyographic signals, especially when the muscles might be of asymmetric size, and have asymmetric fiber-type distributions. ${ }^{16}$ Electromyography has provided somewhat contradictory evidence as to the changes in muscle activity with brace wearing. ${ }^{17,18}$ Wynarsky and Schultz ${ }^{19}$ used an optimization approach to identify muscle activation patterns that could reduce the magnitude of a scoliosis deformity (as opposed to the spinal loading strategies investigated here). They found that spinal curvature and displacement could be reduced, but that the amount of correction was limited by the muscle activation intensity that could be achieved. 
One can speculate that different individuals with scoliosis adopt differing muscle activation strategies and that some strategies predispose to a spinal loading state that produces deformity progression during growth, while others do not. These simulations demonstrate that there are multiple plausible loading states that would result in a wide range of lateral bending moments in the motion segments, hence differing degrees of asymmetric loading of the vertebrae and discs. Some loading conditions might cause scoliosis progression, while others would not. The muscle strategies that appear to protect the spine from loads that are likely to cause deformity progression require a greater level of muscle activation, including antagonistic activation, with associated physiologic costs of increased muscle energy expenditure and overall spinal loading.

\section{- Key Points}

- Asymmetric loading of the skeletally immature spine with scoliosis is thought to produce asymmetric spinal development and hence progressive deformity. - A biomechanical analysis of three differing muscle activation strategies indicated that the spine's concave side could be more or less loaded than the convex side, depending on the strategy.

- The findings permit speculation that the probability of scoliosis progression would depend on an individual's muscle activation strategy, and suggest that conservative management could influence the mechanism of progression.

\section{References}

1. Roaf R. Vertebral growth and its mechanical control. J Bone Joint Surg Br 1960;42:40-59.
2. Stokes IAF. Analysis of symmetry of vertebral body loading consequent to lateral spinal curvature. Spine 1997;22:2495-503.

3. Villemure I, Aubin CE, Dansereau J, et al. Simulation of progressive deformities in adolescent idiopathic scoliosis using a biomechanical model integrating vertebral growth modulation. J Biomech Eng 2002;124:784-90.

4. Little DG, Song KM, Katz D, et al. Relationship of peak height velocity to other maturity indicators in idiopathic scoliosis in girls. J Bone Joint Surg Am 2000;82:685-93.

5. Stokes IAF, Gardner-Morse MG. Lumbar spine maximum efforts and muscle recruitment patterns predicted by a model with multijoint muscles and joints with stiffness. J Biomech 1995;28:173-86.

6. Stokes IAF, Gardner-Morse M. Quantitative anatomy of the lumbar musculature. J Biomech 1999;32:311-6.

7. Gardner-Morse MG, Stokes IAF. Structural behavior of human lumbar spinal motion segments. J Biomech 2004;37:205-12.

8. Stokes IA, Gardner-Morse M. Spinal stiffness increases with axial load: another stabilizing consequence of muscle action. J Electromyogr Kinesiol 2003;13:397-402.

9. Hughes RE, Chaffin DB, Lavender SA, et al. Evaluation of muscle force prediction models of the lumbar trunk using surface electromyography. J O $r$ thop Res 1994;12:689-98.

10. Patwardhan AG, Havey RM, Meade KP, et al. A follower load increases the load-carrying capacity of the lumbar spine in compression. Spine 1999;24: 1003-9.

11. Perié D, Sales de Gauzy J, Curnier D, et al. Intervertebral disc modeling using a MRI method: migration of the nucleus zone within scoliotic intervertebral discs. Magn Reson Imaging 2001;19:1245-8.

12. Urban MR, Fairbank JC, Bibby SR, et al. Intervertebral disc composition in neuromuscular scoliosis: changes in cell density and glycosaminoglycan concentration at the curve apex. Spine 2001;26:610-7.

13. Antoniou J, Arlet V, Goswami T, et al. Elevated synthetic activity in the convex side of scoliotic intervertebral discs and endplates compared with normal tissues. Spine 2001;26:E198-206.

14. Bibby SR, Fairbank JC, Urban MR, et al. Cell viability in scoliotic discs in relation to disc deformity and nutrient levels. Spine 2002;27:2220-8.

15. Urban MR, Fairbank JCT, Etherington PJ, et al. Electrochemical measurement of transport into scoliotic intervertebral discs in vivo using nitrous oxide as a tracer. Spine 2001;26:984-90.

16. Mannion AF, Meier M, Grob D, et al. Paraspinal muscle fibre type alterations associated with scoliosis: an old problem revisited with new evidence. Eur Spine J 1998;7:289-93.

18. Odermatt D, Mathieu PA, Beauséjour M, et al. Electromyography of scoliotic patients treated with a brace. J Orthop Res 2003;21:931-6.

19. Wynarsky GT, Schultz AB. Trunk muscle activities in braced scoliosis patients. Spine 1989;14:1283-6. 